\title{
Evaluating the Clinical Utility of Noninvasive Methods for Measuring Tear Film Surface Quality
}

\author{
Dorota H. Szczesna, ${ }^{* 1}$ D. Robert Iskander, ${ }^{2}$ and Henryk T. Kasprzak ${ }^{1}$ \\ ${ }^{1}$ Institute of Physics, Wroclaw University of Technology, Wybrzeże Wyspiańskiego 27, 50-370 Wroclaw, \\ ${ }^{2}$ Institute of Biomedical Engineering and Instrumentation, Wroclaw University of Technology,
} Plac Grunwaldzki 13, 50-377 Wrocław

Received February 16, 2011; accepted March 11, 2011; published March 31, 2011

\begin{abstract}
The cornea is covered by a thin tear film that performs various vital functions including the provision of a high quality optical surface and protecting the cornea. Clinical methods for assessing tear film surface quality are mostly invasive and often unreliable. Recently, several non-invasive methods have been proposed. In this letter, we review three promising optical techniques based on high speed videokeratoscopy, wavefront sensing and lateral shearing interferometry, and evaluate their clinical utility.
\end{abstract}

The tear film is the first optical element of the human eye. At the air/tear film interface the rays undergo the strongest refraction (from about $n=1$ to about $n=1.336$ ). Hence, the stability of the pre-corneal tear film surface and its smoothness are essential for the retinal image to have a good quality [1]-[3]. The tear film is broadly comprised of three distinct layers, an outer lipid layer, a middle aqueous layer and an inner mucin layer. The tear film is rejuvenated with every blink and will eventually rupture if blinking does not occur. Instability or rupture of the tear film leads to the collection of symptoms known as dry eye, one of the most commonly reported eye ailments [4].

Currently, clinically accepted assessment of tear film often includes patient history, slit-lamp biomicroscope examination of corneal and conjunctival staining with vital dyes, fluorescein tear break-up time, subjective assessment of meibomian gland secretions, conjunctival hyperaemia, and evaluation of tear film volume with a Schirmer test, cotton thread test, or tear meniscus height. However, such a battery of diagnostic methods is time consuming and may be unreliable [5], [6]. Also, such methods are inadequate to study the kinetics of tear film and their influence on visual performance of the eye.

In the last decade, more attention was given to the developments of non-invasive methods for pre-corneal tear film surface analysis such as repeated measure videokeratography [7], [8], high-speed videokeratoscopy [9]-[11], dynamic wavefront sensing [12], [13] and interferometry [14]-[18]. There are also attempts to combine the techniques as in the case of wavefront sensing and retroillumination [19].

\footnotetext{
*E-mail: dorota.szczesna@pwr.wroc.pl
}

Our team has pioneered two of those techniques, namely lateral shearing interferometry [17], [18], [20], [21], and high-speed videokeratoscopy [9], [10]. We have also been actively involved in the development of wavefront sensing methodologies [13], [22] for the assessment of tear film in the human eye.

In the lateral sharing interferometer, the wavefront coming from a HeNe laser reflects from the tear film surface and creates an interferogram. The shape of interference fringes corresponds to a temporal stage of the precorneal tear film surface. The method of numerical analysis is based on combined image processing techniques and spectral analysis of the interferogram [21], [23].

The high-speed videokeratoscopy method is based on the projection of a Placido disc pattern onto the precorneal tear film layer, and capturing the reflection with a video-camera. Over time, the quality of the reflected image, in particular the image coherence, provides a timevarying tear film surface quality indicator [9]-[11].

For dynamic wavefront sensing, a commercially available Shack-Hartmann wavefront sensor was used (COAS $^{\mathrm{TM}}$ WaveFront Sciences Inc.). Wavefront aberrations were fitted with a series of Zernike polynomials and rescaled to the smallest common pupil diameter in each of the recordings [24]. The wavefront dynamics measurements represent time-series of each Zernike aberration component up to a certain radial order, as well as the Zernike polynomial RMS fit error. Other time-varying parameters, such as refractive measures or image quality matrices [25], [26] can also be derived.

Representative examples of data acquired from the three considered instruments from a subject diagnosed with dry eye are shown in Fig. 1, when the quality of tear film is good (left column) and after a tear film breakup was detected (right column). Since the raw Shack-Hartmann images of lenslet spots are difficult to distinguish, corresponding refractive power maps (in dioptres) of higher order aberrations are shown. 

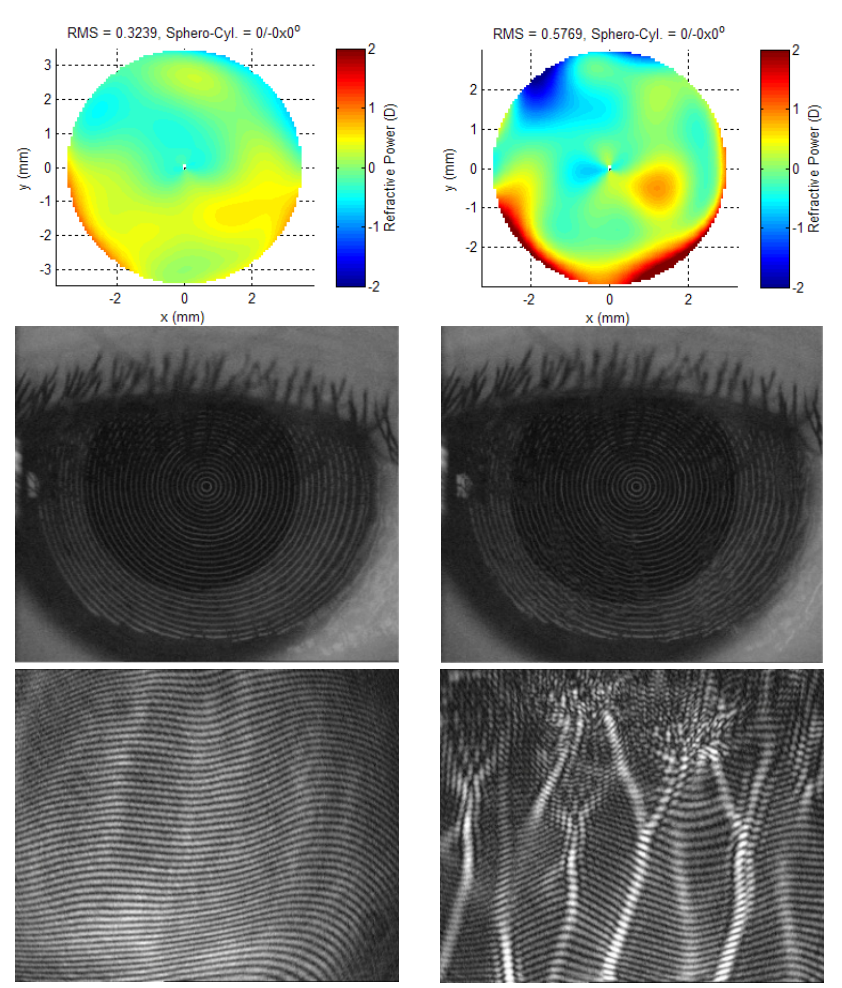

Fig. 1. Representative examples of data for a subject diagnosed with dry eye. Refractive power maps from a Shack-Hartmann sensor (top row), high-speed videokeratoscopy images (centre row), and lateral shearing interferograms (bottom row) for good tear film surface (left column) and after a detected tear film breakup (right column). Note that the three sets of data have not been acquired simultaneously.

Recently, all three techniques have been rigorously compared in a clinical study involving 18 healthy subjects [27], and a study involving 22 healthy and 12 subjects clinically diagnosed with dry eye [28]. It was found that among those methods high-speed videokeratoscopy was the most precise in measuring tear film surface quality while lateral shearing interferometry was the most sensitive method for analyzing the tear film build-up phase. In fact, it is the only method that is able to distinguish up to five phases of tear film surface kinetics [29]. For detecting dry eye, the interferometric technique showed the best detection performance (AUC [area under the $\mathrm{ROC}]=0.80$ in suppressed blinking conditions and 0.73 in natural blinking conditions), closely followed by high-speed videokeratoscopy $(\mathrm{AUC}=0.72$ and 0.71 , respectively). Wavefront sensing was much less powerful.

The question arises whether the three non-invasive techniques discussed here can be used in a clinical setting. For this, several important factors have to be taken into account. They include:

1. instrument/patient interface,

2. type of light source and type of imaging device,

3. type and amount of input data,

4. patient "chair" time, and

5. ease of operation.
Instrument/patient interface. Imaging techniques used for assessing tear film surface quality require much heavier and robust head-rests than those typically used in ophthalmic instrumentation [30]; bite-bars, however, were found to be unnecessary. All three instruments were noninvasive. In the case of high-speed videokeratoscopy, which was based on a commercially available instrument (Medmont E300, Medmont Pty Ltd., Melbourne, Australia), there was often a light contact between the instrument's cone and the skin in the eye socket. Only few naïve subjects complained during experiments. In the other two techniques, the distance between the patient and the instruments was above $10 \mathrm{~mm}$. The instrument/patient interface was important, particularly during longer data acquisitions. The blinking rate, during natural blinking conditions, was not significantly different between the three instruments, indicating that the instrument/patient interfaces did not significantly influence this mechanism.

Type of light source and type of imaging device. Each of the instruments has a different optical set-up. In the lateral shearing interferometer prototype, an $8 \mathrm{~mW} \mathrm{HeNe}$ laser, $\lambda=632.8 \mathrm{~nm}$, with a $1 \mathrm{~ms}$ shutter was used as an illumination source while a $704 \times 576$ pixel CCD camera was used as an imaging device. The available sampling was $25 \mathrm{fps}$. The amount of light entering the eye was below the safety level of the ANSI 2000 standard [31] and was found to be comfortable for the majority of subjects. No reflex tearing was observed during the measurements. The only problem subjects faced was the difficulty to focus on the target as the stimulus was given to the fellow eye, particularly when the tear film in the dominant eye was tested.

In the high-speed videokeratoscope a narrow Placido disk cone was the illumination source. A $768 \times 572$ pixel CCD camera served as an imaging device. The sampling rate was variable up to $50 \mathrm{fps}$. None of subjects was concerned with the amount of light produced by the instrument and found to easily focus on the internal instrument's target.

As mentioned earlier, for dynamic wavefront sensing, a commercially available $\operatorname{COAS}^{\mathrm{TM}}$ was used. In this system the light source comprises an $840 \mathrm{~nm}$ infrared super-luminescent diode. A $752 \times 480$ (non-square, $9.8 \times 8.4 \mu \mathrm{m})$ pixel $\mathrm{CCD}$ camera served as an imaging device. A square array of $33 \times 44$ lenslets is used in the system. The sampling rate was up to $11 \mathrm{~Hz}$, although, from time to time, significant jitter was encountered, as the system does not have dedicated hardware and is prone to operating system interrupts. As in the case of a highspeed videokeratoscope, no subject found it difficult to undergo the experiment. Few subjects complained about getting their eyes dry at the end of the measurement, particularly during the so called suppressed blinking 
conditions. However, no environmental factors could account for this as all instruments were placed in the same room in which both the temperature and humidity were controlled.

Type and amount of input data. No real-time analysis was available in any of the instruments and all acquired images had to be processed off-line. In essence, all three instruments provide sets of images (roughly of the same resolution) at a given sampling rate. Hence, the amount of basic input data is comparable. However, the computational expense when processing the acquired images is the highest for high-speed videokeratoscopy as it requires advanced image processing techniques. This is followed by lateral shearing interferometry and wavefront sensing.

Patient "chair" time. This is an important factor as the amount of time spent in the clinic using currently available methods to adequately assess tear film surface quality is significant to the point that tear film is not routinely examined in optometry/ophthalmology practices unless there is a patient's complaint. Our studies with the three non-invasive systems indicate that tear film surface quality can be adequately assessed in a series of short measurements of around 10 seconds, in which the subject suppresses the blink or in a one longer measurement, say up to 40 seconds, in which the subject blinks naturally. In diagnosing dry eye, the detection power is higher in suppressed blinking conditions than when the subject blinks naturally. Hence, for the non-invasive methods the "chair" time is significantly shorter than that required in traditional clinical setting.

Ease of operation. Both high-speed videokeratoscopy and dynamic wavefront sensing do not require long subject preparation. In essence, they are commercial instruments so the user interfaces including focusing devices and GUIs have been adequately developed. The lateral shearing interferometer, being the only prototype, requires a little bit more operator preparation for the instrument to find the right focal plane since no automatic focusing/eye tracking device has been implemented yet. This adds to the difficulty of operation as the operator is required to manually track the eye, potentially increasing the inter-observer variability of the method.

Summarising, the three considered non-invasive methods for measuring tear film surface quality have great potential to become clinical instruments. It is easy to envisage high-speed videokeratoscopy soon becoming standard instrumentation in optometrical practice where clinicians will simultaneously examine corneal surface geometry and tear film quality without increasing the "chair" time. Similarly, one can foresee that during routine wavefront dynamic measurements one would also acquire information on tear film kinetics. These are no distant future predictions as the technology is already available. On the other hand, lateral shearing interferometry, despite the fact that it currently prevails in the laboratories, cannot be directly utilised in a clinical practice without further technological advancements. We focus our efforts on improving this very promising technique.

We thank Prof. Michael Collins and the members of the CLVO Lab, Queensland University of Technology, Australia, for their constant support in this project.

\section{References}

[1] N.L. Himebaugh, L.N. Thibos, A. Bradley, G. Wilson, C.G. Begley, Adv. Exp. Med. Biol. 506(Pt B), 1141 (2002).

[2] R. Montés-Micó, J. Cataract. Refract. Surg. 33, 1631 (2007).

[3] R. Tutt, A. Bradley, C. Begley, L.N. Thibos, Invest. Ophthalmol. Vis. Sci. 41, 4117 (2000).

[4] M.A. Lemp, CLAO Journal 21, 221 (1995).

[5] L.S. Mengher, K.S. Pandher, A.J. Bron, Acta Ophthalmol. 64, 441 (1986).

[6] K.K. Nichols, G.L. Mitchell, K. Zadnik, Cornea 23:272 (2004).

[7] J. Nemeth, B. Erdélyi, B. Csákány, P. Gáspár, A. Soumelidis, F. Kahlesz, Z. Lang, Invest. Ophthalmol. Vis. Sci. 43, 1783 (2003).

[8] T. Goto, X. Zheng, S.D. Klyce, H. Kataoka, T. Uno, M. Yamaguchi et al., Am. J. Ophthalmol. 137, 116 (2004).

[9] D.R. Iskander, M. J. Collins, B. Davis, IEEE Trans. Biomed. Eng. 52, 1939 (2005).

[10] D.R. Iskander, M.J. Collins, Clinic. Exp. Optometry 88, 223 (2005).

[11] D. Alonso-Caneiro, D.R. Iskander, M.J. Collins, IEEE Trans. Biomed. Eng. 56, 1473 (2009).

[12] H. Hofer, P. Artal, B. Singer, J.L. Aragón, D.R. Williams, J. Opt. Soc. Am. A 18, 497 (2001).

[13] D.R. Iskander, M.J. Collins, M.R. Morelande, M. Zhu, IEEE Trans Biomed. Eng. 51, 1969 (2004).

[14] A. Dubra, C. Paterson, C. Dainty. Appl. Opt. 43, 1108 (2004).

[15] A. Dubra, C. Paterson, C. Dainty. Appl. Opt. 44, 1191 (2005).

[16] P.E. King-Smith, B.A. Fink, JJ. Nichols, K.K. Nichols, R.M. Hill, J. Opt. Soc. Am. A 23, 2097 (2006).

[17] D.H. Szczesna, H.T. Kasprzak, J. Jaronski, D.A. Rydz, U. Stenevi, Acta Ophthalmol. Scand. 85, 202 (2007).

[18] D.H. Szczesna, H.T. Kasprzak, Ophthalmic Physiol. Opt. 29, 211 (2009).

[19] H. Liu, L.N. Thibos, C.G. Begley, A. Bradley, Invest. Ophthalmol Vis. Sci. 51, 3318 (2010)

[20] T.J. Licznerski, H.T. Kasprzak, W. Kowalik, J. Bio. Opt. 3, 32, (1998).

[21] D.H. Szczesna, J. Jaronski, H.T. Kasprzak, U. Stenevi, J. Bio. Opt. 11, 034028, (2006)

[22] M. Zhu, M.J. Collins, D.R. Iskander. Ophthalmic Physiol. Opt. 26, 439 (2006).

[23] D.H. Szczesna, D.R. Iskander, J. Bio. Opt. 14, 064039 (2009).

[24] J. Schwiegerling, J. Opt. Soc. Am. A 19, 1937 (2002).

[25] D.R. Iskander, B.A. Davis, M.J. Collins, R. Franklin, Ophthal. Physiol. Opt. 27, 245 (2007).

[26] D.R. Iskander, Optom. Vis. Sci. 83, 57, (2006).

[27] D.H. Szczesna, D. Alonso-Caneiro, D.R. Iskander, S.A. Read, M.J. Collins, J. Bio. Opt. 15, 037005 (2010).

[28] D.H. Szczesna, D. Alonso-Caneiro, D.R. Iskander, S.A. Read, M.J. Collins, Invest. Ophthalmol. Vis. Sci. 52, 751 (2011).

[29] D.H. Szczesna, D.R. Iskander, Optom. Vis. Sci. 87, 513 (2010).

[30] H.T. Kasprzak, D.R. Iskander, IEEE Trans. Instrument. Meas. 59, 164 (2010).

[31] F.C. Delori, R.H. Webb, D.H. Sliney, J. Opt. Soc. Am. A 24, 1250 (2007) 